\title{
Evaluation of the Respiratory Muscular Function by means of Diaphragmatic Mechanomyographic Signals in COPD patients
}

\author{
Leonardo Sarlabous, Abel Torres, Member, IEEE, José A. Fiz, \\ Joaquim Gea, Juana M. Martínez-Llorens, and Raimon Jané, Member, IEEE
}

\begin{abstract}
The study of mechanomyographic (MMG) signals of respiratory muscles is a promising technique in order to evaluate the respiratory muscular effort. In this work MMG signals from left and right hemidiaphragm (MMGI and MMGr, respectively) acquired during a respiratory protocol have been analyzed. The acquisition of both MMG signals was carried out by means of two capacitive accelerometers placed on both left and right sides of the costal wall. The signals were recorded in a group of six patients with Chronic Obstructive Pulmonary Disease (COPD). It has been observed that with the increase of inspiratory pressure it takes place an increase of the amplitude and a displacement toward low frequencies in both left and right MMG signals. Furthermore, it has been seen that the increase of amplitude and the decrease of frequency in MMG signals are more pronounced in severe COPD patients. This behaviour is similar for both MMGI and MMGr signals. Results suggest that the use of MMG signals could be potentially useful for the evaluation of the respiratory muscular function in COPD patients.
\end{abstract}

\section{INTRODUCTION}

$\mathrm{D}$ URING muscular contraction, besides shortening and/or force production, a transversal movement (perpendicular to muscle fibers direction) takes place. This movement is produced by lateral expansion of the active muscle fibers and can be decomposed into two parts, according to the movement type: (1) a low frequency movement that takes place mainly during the beginning and the end of muscle contraction in isometric contractions, and in general during the whole contraction in dynamic contractions (LF component), (2) a high frequency movement that consists on small oscillations or vibrations that take place during the whole contraction (HF component). This second kind of movement is usually denominated mechanomyogram (MMG) [1].

Both movements could be acquired by means of different non invasive sensors (air coupled microphones, piezoelectric contact sensors and accelerometers) fixed on the surface of

This work was supported in part by grants from Ministerio de Ciencia y Innovación of Spain (TEC2007-68076-C02-01) and in part by the Ministerio de Asuntos Exteriores y de Cooperación (AECID scholarship)

L. Sarlabous, A. Torres and R. Jané are with Dept. ESAII, Universitat Politècnica de Catalunya, Institut de Bioenginyeria de Catalunya (IBEC) and CIBER de Bioingeniería, Biomateriales y Nanomedicina (CIBERBBN), Barcelona, Spain (e-mail: leonardo.sarlabous@upc.edu, abel.torres@upc.edu, raimon.jane@upc.edu).

J. A. Fiz is with the Department of Pneumologie of the Navarra Hospital, IBEC and CIBER-BBN, Pamplona, Spain (e-mail: jfizfern@navarra.es).

J. Gea and J. M. Martínez-Llorens are with the Department of Respiratory Medicine at the IMIM- Hospital del Mar, UPF, CIBERES, Barcelona, Spain (e-mail: jgea@imim.es, jmartinezl@imim.es). the skin, over the muscle belly. It has been observed that the amplitude of both LF contraction components [2]-[5] and HF contraction components [1], [6]-[8] of the muscular movement increase with the contraction force. The rationale for inferring the contraction muscle force, from the amplitude of the LF and HF components of the muscle movement, is that the signal constitutes the summation of muscle fibers movement from the recruited muscle motor units and their firing rate.

The respiratory muscular fatigue can be monitored by means of the implementation of techniques that study the muscular electric activity in amplitude and frequency through the electromyographic (EMG) signal of respiratory muscles. The diaphragm is the main respiratory muscle responsible for the mechano-respiratory activity. The main inconvenience of the utilization of the diaphragmatic EMG signal is that it is needed a complex and invasive instrumentation for its realization (esophageal electrode catheter). On the other hand, the use of surface electrodes has great inconveniences due to the contamination of bioelectric noise coming from other origins, making its application questionable.

In patients with Chronic Obstructive Pulmonary Disease (COPD) of type A (lung emphysema), the respiratory muscular function and the mechanic of the thoracic cage are very affected. The muscular efficiency (relationship between electric and mechanic activities of the muscle) is very reduced, due to the changes in the thoraco-diaphragmatic space configuration produced by the disease, and that makes ineffective the muscular contraction, wasting great amounts of energy.

In this work it is introduced a new method for monitoring the respiratory muscular activity and the dynamic of the thoracic cage, applied to patients with COPD. This new method is based on the study of the MMG signal of the diaphragm muscle in order to analyze the mechanical activity of this muscle in connection with the developed respiratory muscular effort.

In previous works [9]-[13], our group has analyzed, in an animal model (dogs), the signal acquired by means of a capacitive accelerometer placed on the costal wall of the thoracic cage in order to register the mechanomyographic (MMG) signal of the diaphragm muscle. In these works, a positive correlation has been found between amplitude parameters of the diaphragmatic MMG signal and the respiratory effort assessed by means of the inspiratory 
TABLE I

PATIENTS CHARACTERISTICS AND PARAMETERS OF THE PULMONARY FUNCTION TESTS

\begin{tabular}{ccccccccc}
\hline \hline & Age (years) & Height $(\mathrm{cm})$ & Weight $(\mathrm{kg})$ & FEV $_{1} \%$ ref. & FVC \% ref. & \% $_{\text {FEV }} /$ FVC & DLco \% ref. & Kco \% ref. \\
\hline EPOC1 & 51 & 166 & 53 & 18 & 49 & 28.63 & 18 \\
EPOC2 & 65 & 162 & 58 & 24 & 42 & 41.17 & 38 \\
EPOC3 & 75 & 167 & 72 & 36 & 52 & 48.62 & 42 \\
EPOC4 & 74 & 164 & 45 & 35 & 56 & 43.66 & 44 \\
EPOC5 & 67 & 173 & 77 & 22 & 43 & 34.63 & 42 & 56 \\
EPOC6 & 77 & 166 & 79 & 44 & 62 & 49.41 & 63 \\
Me \pm STD & $68.2 \pm 9.6$ & $166.3 \pm 3.7$ & $64.0 \pm 14.0$ & $29.8 \pm 10.0$ & $50.7 \pm 7.7$ & $41.0 \pm 8.1$ & $41.2 \pm 14.4$ & $57.3 \pm 10.7$ \\
\hline \hline
\end{tabular}

$\mathrm{FEV}_{1}=$ forced expiratory volume over one second, $\mathrm{FVC}=$ forced vital capacity, $\mathrm{FEV}_{1} / \mathrm{FVC}=$ proportion of the forced vital capacity exhaled in the first second, $\mathrm{DLco}=$ carbon monoxide diffusing capacity, $\mathrm{Kco}=$ carbon monoxide transfer coefficient $\%$ ref $=$ percentage regarding the predicted value, $\mathrm{Me}=$ mean, $\mathrm{STD}=$ standard deviation .

pressure. The objective of the present work has been to corroborate and extrapolate these results to human experiments and to analyze differences between the developed mechanical activity in function of the severity of the pulmonary obstruction in patients with COPD.

\section{Methodology}

\section{A. Study population}

The study population consisted of six patients with type A COPD, with smoking antecedents and diagnosed by means of forced spirometry and TAC illustrative of lung emphysema. Table I reports the clinical characteristics and the parameters of the pulmonary function tests of this group of COPD patients.

\section{B. Signals and instrumentation}

The subjects were instrumented in order to acquire three biomedical signals: the inspiratory pressure (IP) and the mechanomyographic signals of the left and right hemidiaphragms (MMGl and MMGr, respectively). IP was measured with a pressure transducer placed in the tube through which the patients breathed. MMGl and MMGr were acquired with two Kistler 8312B2 capacitive accelerometers placed on the surface of the thoracic cage. The placement of the sensors (between the seventh and eighth intercostal spaces in the left and right anterior axillary lines) was chosen with the intention of obtaining the mechanomyographic signal of both left and right hemidiaphragms.

All analog signals were amplified, analog filtered, digitized with a 12 bit A/D system at a sampling rate of 4 $\mathrm{kHz}$, and decimated at a new sampling rate of $200 \mathrm{~Hz}$.

\section{Respiratory protocol}

The six COPD patients performed a progressive incremental flow protocol. During the respiratory protocol the subject is seated and breathes without respiratory assistant through the mouth by means of a mouthpiece and a tube, and with a nose clip. During the realization of the respiratory tests, the medical personnel instruct to the patients in order to perform correctly the protocol. The test begins with quiet respiration at basal tidal volume and then the rhythm and intensity of the breathing is increased progressively until the patients carried out several respiratory cycles at their vital capacity. Later the rhythm an intensity of the breathing is decreased progressively until quiet respiratory breathing at basal tidal volume is reached again. This process is repeated two or three times for each patient. The approximate duration of these respiratory tests is between two and three minutes. The increment of the rhythm and the breathing intensity causes an increase of the inspiratory pressure and of the respiratory muscular effort. This increase in the respiratory muscular effort can be analyzed by means of the MMG signals.

Fig. 1 shows an example of the IP, MMGl and MMGr signals corresponding to a progressive incremental flow protocol. As it can be seen, during the test the respiratory effort (assessed by means the inspiratory pressure) varies considerably.

The duration, number of respiratory cycles and maximum inspiratory pressure reached during the segments with incremental flow of the respiratory test by each patient are shown in Table II.

\section{Signal Processing}

Identification of respiratory cycles and detection of initial and final time of diaphragm muscle contraction was made by means of the IP signal. Two IP signal parameters were estimated: the mean ( $\left.\mathrm{IP}_{\text {mean }}\right)$ and maximum $\left(\mathrm{IP}_{\max }\right)$ inspiratory pressure achieved during the respiratory cycle.

TABLE II

DURATION, NUMBER OF CYCLES AND MAXIMUM INSPIRATORY PRESSURE DEVELOPED IN THE RESPIRATORY TESTS

\begin{tabular}{cccc}
\hline \hline & Duration (s) & $\begin{array}{c}\text { Number of } \\
\text { cycles }\end{array}$ & $\begin{array}{c}\text { Maximum IP } \\
(\mathrm{cmH} 2 \mathrm{O})\end{array}$ \\
\hline EPOC1 & 89 & 82 & 3.0 \\
EPOC2 & 130 & 54 & 3.5 \\
EPOC3 & 102 & 102 & 4.2 \\
EPOC4 & 71 & 36 & 3.9 \\
EPOC5 & 112 & 53 & 2.9 \\
EPOC6 & 95 & 67 & 4.3 \\
\hdashline Me \pm STD & $99.8 \pm 20.2$ & $65.7 \pm 23.5$ & $3.6 \pm 0.6$ \\
\hline \hline
\end{tabular}



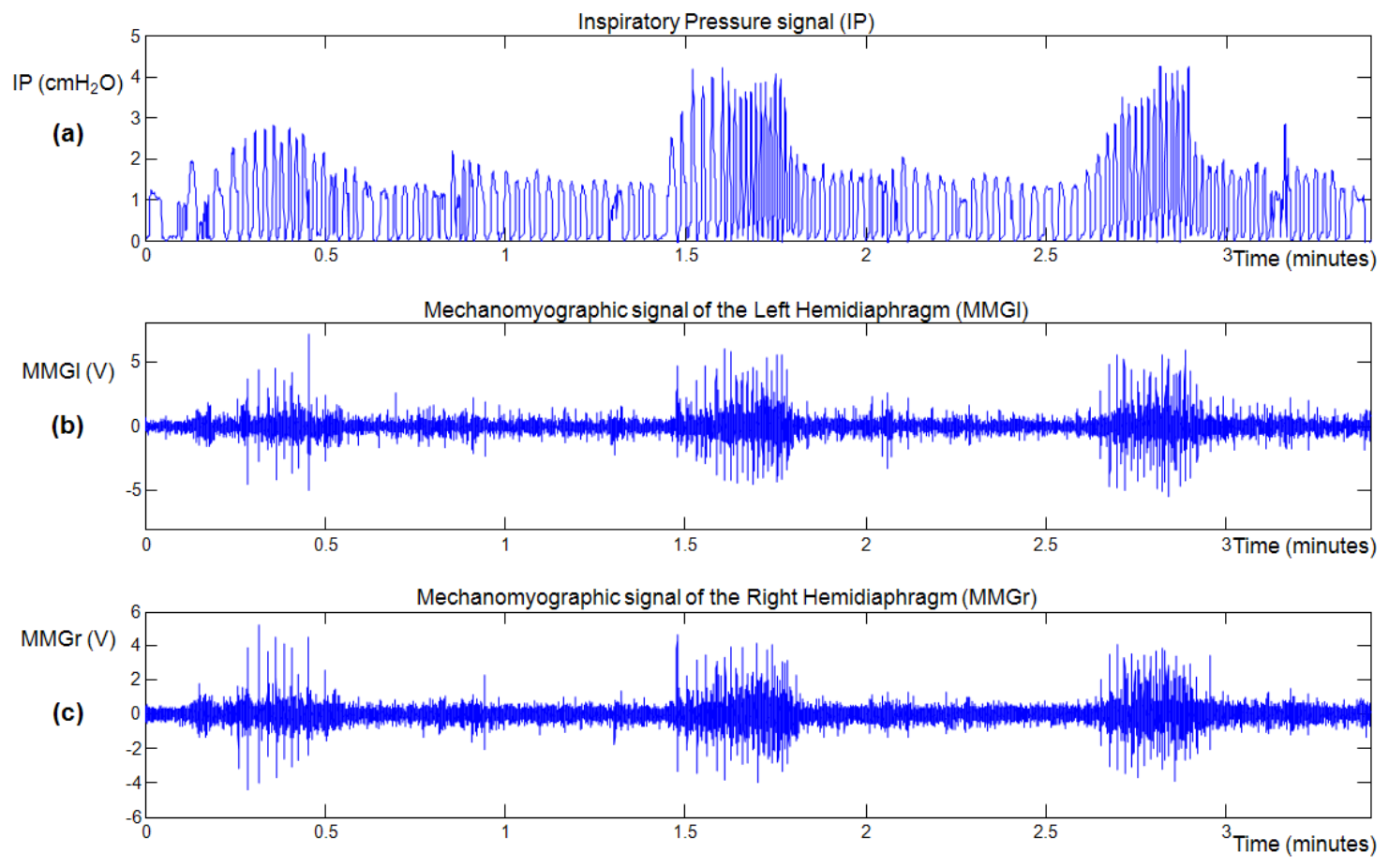

Fig. 1. Example of signals acquired during the progressive incremental flow respiratory test: (a) Inspiratory pressure (IP) signal, (b) left diaphragmatic mechanomyographic (MMGl) signal, (c) right diaphragmatic mechanomyographic (MMGr) signal

In order to quantify the changes in amplitude and frequency in the MMG signals, four parameters were estimated in both MMGl and MMGr signals:

- the root mean square (RMS)

- the Rényi entropy with $\alpha=0.5(\mathrm{H})$

- the Multistate Lempel-Ziv coefficient with 500 states (LZM)

- the Maximum Frequency (fmax) (95\% of the energy of the spectrum)

The explanation of the Rényi entropy and MLZ estimation techniques are detailed in [14] and [15], respectively.

The relationship between the IP parameters $\left(\mathrm{IP}_{\text {mean }} \mathrm{y}\right.$ $\left.\mathrm{IP}_{\max }\right)$ and $\mathrm{MMG}$ parameters (RMS, H, LZ and fmax) were analyzed by means of the Pearson correlation coefficient.

\section{RESULTS}

Correlation coefficients between the maximum inspiratory pressure developed $\left(\mathrm{IP}_{\max }\right)$ and the diaphragmatic $\mathrm{MMG}$ parameters (RMS, H, MLZ and fmax) for the six analyzed COPD patients are shown in Table III.
Positive high correlation coefficients were found for MMG amplitude parameters whereas negative correlation coefficients were found for MMG maximum frequency. That means that when the IP increases an augment of amplitude and a displacement towards lower frequencies takes place in the MMG signals. This behavior is observed in a similar way in both hemidiaphragms (MMGl and MMGr).

As for the performance of the three analyzed MMG amplitude parameters (RMS, H and MLZ), the entropy parameter $(\mathrm{H})$ is the one with better average, although in the MMGl the performance of the MLZ parameter is practically identical and with smaller standard deviation. In general, the behavior of the RMS parameter is inferior to the behavior of $\mathrm{H}$ parameter.

Table IV shows the correlation coefficients between the slope of the evolution of MMG parameters with the increment of IP, and the pulmonary function test parameters (FEV ${ }_{1} \%$ ref., $\mathrm{FVC} \%$ ref.,\% $\mathrm{FEV}_{1} / \mathrm{FVC}, \mathrm{DLco} \%$ ref., and $\mathrm{Kco} \%$ ref.) for the six COPD patients.

TABLE III

CORRELATION COEFFICIENTS BETWEEN INSPIRATORY PRESSURE AMPLITUDE AND THE MMG PARAMETERS

\begin{tabular}{|c|c|c|c|c|c|c|c|c|}
\hline & \multicolumn{4}{|c|}{ Left MMG } & \multicolumn{4}{|c|}{ Right MMG } \\
\hline & RMS & $\mathrm{H}$ & MLZ & fmax & RMS & $\mathrm{H}$ & MLZ & fmax \\
\hline COPD1 & 0.785 & 0.820 & 0.795 & -0.560 & 0.819 & 0.818 & 0.786 & -0.490 \\
\hline COPD2 & 0.767 & 0.832 & 0.861 & -0.145 & 0.334 & 0.369 & 0.011 & -0.667 \\
\hline COPD3 & 0.575 & 0.589 & 0.593 & -0.114 & 0.625 & 0.647 & 0.655 & -0.601 \\
\hline COPD4 & 0.673 & 0.689 & 0.697 & +0.508 & 0.761 & 0.790 & 0.724 & -0.291 \\
\hline COPD5 & 0.831 & 0.878 & 0.854 & -0.639 & 0.823 & 0.835 & 0.713 & -0.719 \\
\hline COPD6 & 0.923 & 0.889 & 0.893 & -0.520 & 0.921 & 0.911 & 0.900 & -0.651 \\
\hline $\mathrm{Me} \pm \mathrm{STD}$ & $0.759 \pm 0.122$ & $0.783 \pm 0.119$ & $0.782 \pm 0.116$ & $-0.245 \pm 0.423$ & $0.714 \pm 0.210$ & $0.728 \pm 0.196$ & $0.632 \pm 0.315$ & $-0.570 \pm 0.157$ \\
\hline
\end{tabular}

RMS = Root Mean Square, H = Rényi entropy, MLZ = Multistate Lempel-Ziv, fmax = Maximum frequency, Me = mean, STD = standard deviation. 
TABLE IV

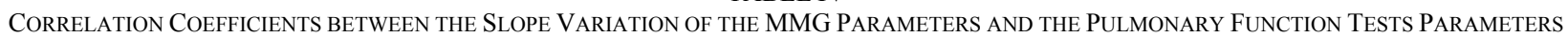

\begin{tabular}{|c|c|c|c|c|c|c|c|c|}
\hline & \multicolumn{4}{|c|}{ Left MMG } & \multicolumn{4}{|c|}{ Right MMG } \\
\hline & RMS & $\mathrm{H}$ & MLZ & fmax & RMS & $\mathrm{H}$ & MLZ & fmax \\
\hline $\mathrm{FEV}_{1} \%$ ref. & -0.65 & -0.77 & -0.74 & 0.50 & -0.21 & -0.11 & -0.06 & 0.61 \\
\hline FVC \% ref. & -0.69 & -0.47 & -0.49 & 0.18 & -0.26 & 0.06 & 0.08 & 0.69 \\
\hline$\% \mathrm{FEV}_{1} / \mathrm{FVC}$ & -0.43 & -0.78 & -0.74 & 0.54 & 0.02 & -0.05 & 0.02 & 0.41 \\
\hline DLco \% ref. & -0.77 & -0.97 & -0.93 & 0.81 & -0.47 & -0.52 & -0.46 & 0.57 \\
\hline Kco \% ref. & -0.69 & -0.81 & -0.77 & 0.40 & $\begin{array}{r}-0.09 \\
-\end{array}$ & -0.17 & -0.18 & 0.07 \\
\hline $\mathrm{Me} \pm \mathrm{STD}$ & $-0.65 \pm 0.13$ & $-0.76 \pm 0.18$ & $-0.73 \pm 0.16$ & $0.49 \pm 0.23$ & $-0.20 \pm 0.19$ & $-0.16 \pm 0.21$ & $-0.12 \pm 0.21$ & $0.47 \pm 0.25$ \\
\hline
\end{tabular}

RMS = Root Mean Square, H = Rényi entropy, MLZ = Multistate Lempel-Ziv, fmax = Maximum frequency, FEV , $_{1}$ forced expiratory volume over one second, $\mathrm{FVC}=$ forced vital capacity, $\mathrm{FEV}_{1} / \mathrm{FVC}=$ proportion of the forced vital capacity exhaled in the first second, DLco = carbon monoxide diffusing capacity, $\mathrm{Kco}=$ carbon monoxide transfer coefficient $\% \mathrm{ref}=$ percentage regarding the predicted value, $\mathrm{Me}=$ mean, $\mathrm{STD}=$ standard deviation .

As it can be seen, the correlation coefficients are negative for MMG amplitude parameters. That means that the more obstructive severity of the COPD patient (a smaller value in the pulmonary function test parameters), the more increment takes place in the MMG signal amplitude (for the same increment of IP). This behavior is similar in both MMGl and MMGr signals.

As for the performance of the pulmonary function test parameters, the capacity of diffusion of monoxide of carbon parameters (DLco\% ref. and Kco\% ref.) are the ones with better correlation coefficients, although a high correlation is also obtained with the parameters of forced expired volume in one second ( $\mathrm{FEV}_{1} \%$ ref.) and the forced vital capacity (FVC\% ref.).

\section{CONCLUSIONS}

In this work diaphragmatic MMG signals have been analyzed, acquired during a respiratory protocol of short duration in which is not necessary to achieve high levels of inspiratory pressure, in such a way that the test is easy to carry out for a patient with obstructive respiratory problems as the COPD patients.

It has been observed that the small increments of inspiratory pressure that take place during the tests (always smaller than $5 \mathrm{cmH} 2 \mathrm{O}$ ) are correlated with increases of amplitude and displacements toward low frequencies in the respiratory muscles MMG signals.

Furthermore, the amplitude and frequency variations in the MMG signals are more pronounced in severe COPD patients. This behavior is presented in a more marked way in the parameters of diffusion of monoxide of carbon (DLco\% ref. and $\mathrm{Kco} \%$ ref.) that are indeed functional markers of the grade of lung emphysema in these COPD patients.

The technique presented in this paper provides an indirect method to assess the respiratory effort by means of MMG signals of the diaphragm muscle. We feel that in the future this technique could provide a new method to study the mechanical properties of respiratory muscles and will be potentially useful for the assessment of the respiratory muscular function in COPD patients.

\section{REFERENCES}

[1] C. Orizio, "Muscle Sound: bases for the introduction of a mechanomyogaphic signal in muscle studies," Crit. Rev. Biomed. Eng., 21, pp. 201-243, 1993

[2] M. Petitjean, B. Maton, and J.-C. Cnockaert, "Evaluation of human dynamic contraction by phonomyography", J. Appl. Physiol., 73, pp. 2567-2573, 1992

[3] D. B. Smith, T. J. Housh, G. O. Johnson, T. K. Evetovixh, K. T. Ebersole, and S. R. Perry, "Mechanomyographic and electromyographic responses to eccentric and concentric isokinetic muscle actions of the biceps brachii", Muscle \& Nerve, 21, pp. 14381444, 1998

[4] J. Celichowski, K. Grottel, and E. Bichler, "Relationship between mechanomyogram signals and changes in force of human forefinger flexor muscles during voluntary contraction", Eur. J. Appl. Physiol., 78, pp. 283-288, 1998

[5] C. Orizio, R. V. Baratta, B. He Zhou, M. Solomonow, and A. Veicsteinas, "Force and surface mechanomyogram frequency responses in cat gastrocnemious", J. Biomech., 33, pp. 427-433, 2000

[6] M. J. Stokes and P. A. Dalton, "Acoustic myographic activity increases linearly up to maximal voluntary isometric force in the human quadriceps muscle,” J. Neurol. Sci., 101, pp.163-167, 1991.

[7] F. Esposito, D. Malgrati, A. Veicsteinas and C. Orizio, "Time and frequency domain analysis of electromyogram and soundmyogram in the elderly,” Eur. J. Appl. Physiol., 73, pp.503-510, 1996.

[8] G. O. Matheson, L. Maffey-Ward, M. Mooney, K. Ladly, K. Fung and Y. Zhang, "Vibromyography as a quantitative measure muscle force production," Scand. J. Rehabil. Med., 29, pp.29-35, 1997.

[9] A. Torres, J.A. Fiz, J. Morera, A.E. Grassino, and R. Jané, "NonInvasive Measurement of Diaphragmatic Contraction Time in Dogs," 23th Ann. Conf. IEEE-EMBS, 2001.

[10] A. Torres, J.A. Fiz, J. Morera, A.E. Grassino, and R. Jané, "TimeFrequency representations of the diaphragmatic movement measured by a surface piezoelectric contact sensor in dogs," 25th Ann. Conf. IEEE-EMBS, 2003.

[11] A. Torres, J.A. Fiz, B. Galdiz, J. Gea, and R. Jané, "Non invasive assessment of respiratory muscle effort by means of the study of diaphragm movement registered with surface sensors. Animal model (dogs)," 26th Ann. Conf. IEEE-EMBS, 2004

[12] A. Torres, J.A. Fiz, B. Galdiz, J. Gea, J. Morera and R. Jané, "A Wavelet Multiscale Based Method to Separate the High and Low Frequency Components of Mechanomyographic Signals," 27th Ann. Conf. IEEE-EMBS, 2005

[13] A. Torres, J.A. Fiz, B. Galdiz, J. Gea, J. Morera and R. Jané, "Inspiratory Pressure Evaluation by means of the Entropy of Respiratory Mechanomyographic Signals," 28th Ann. Conf. IEEEEMBS, 2006

[14] A. Torres, J.A. Fiz, R. Jané, E. Laciar, B. Galdiz, J. Gea and J. Morera, "Rényi Entropy and Lempel-Ziv Complexity of Mechanomyographic Recordings of Diaphragm Muscle as Indexes of Respiratory Effort," 30th Ann. Conf. IEEE-EMBS, 2008

[15] L. Sarlabous, A. Torres, J.A. Fiz, J. Gea, B. Galdiz and R. Jané, "Multistate Lempel-Ziv Index Interpretation as a Measure of Amplitude and Complexity Changes," 31th Ann. Conf. IEEE-EMBS, 2009 